\title{
EL MEDIO COMO ESPACIO EDUCATIVO EN TIEMPOS DE CRISIS: EVALUACIÓN DE UNA EXPERIENCIA FUERA DEL AULA Y SU APLICACIÓN, LA TRANSMISIÓN DE ACTITUDES, VALORES Y CONOCIMIENTOS RELACIONADOS CON LA ENSEÑANZA Y EL APRENDIZAJE DE LAS GEOCIENCIAS.
}

\author{
Eduardo Rebollada Casado \\ Facultad de Formación del Profesorado de la Universidad de Extremadura \\ Avda. de la Universidad s/n. 10071 Cáceres \\ erebolla@alumnos.unex.es \\ José María Corrales Vázquez \\ Facultad de Formación del Profesorado de la Universidad de Extremadura \\ Avda. de la Universidad s/n. 10071 Cáceres \\ corrales@unex.es
}

Recepción Artículo: 5 noviembre 2020 Admisión Evaluación: 5 noviembre 2020 Informe Evaluador 1: 12 noviembre 2020 Informe Evaluador 2: 17 noviembre 2020 Aprobación Publicación: 27 noviembre 2020

\section{RESUMEN}

Desde hace algo más de un año la pandemia COVID-19 viene produciendo un impacto global que ha tenido especial repercusión en aspectos sanitarios y educativos. En países como el nuestro se han visto alterados los procesos de enseñanza y aprendizaje en todos los niveles educativos.

Ante esta situación extraordinaria, el sistema educativo español ha tratado de adaptarse a la nueva coyuntura caracterizada por las restricciones para amortiguar los efectos de la pandemia, propiciando en ocasiones estrategias de enseñanza novedosas, como el uso casi exclusivo de las TIC en una situación de excepcional durante más de medio curso escolar. En aquellas disciplinas científicas donde son comunes las salidas fuera del aula, sin embargo, se han estado produciendo además cambios constantes en los procedimientos de trabajo, necesarios igualmente para adecuar la enseñanza al nuevo statu quo.

En algunas disciplinas científicas donde es habitual la utilización de los laboratorios y las salidas fuera de las aulas se sufrió de manera especial la pandemia durante el curso 2019-20. Sin embargo, durante el último curso, las medidas especiales de enseñanza con una ventilación adecuada ha propiciado la realización de salidas fuera del aula en asignaturas relacionadas con la Biología y Geología, basadas en intervenciones educativas en el medio.

El resultado ha sido una imagen que nos permite presentar en este trabajo la comparativa de los datos obtenidos tras el análisis de las impresiones sobre la participación de 648 alumnos y alumnas de dieciocho centros 


\section{EL MEDIO COMO ESPACIO EDUCATIVO EN TIEMPOS DE CRISIS: EVALUACIÓN DE UNA EXPERIENCIA FUERA DEL AULA Y SU APLICACION, LA TRANSMISION DE ACTITUDES, VALORES Y CONOCIMIENTOS RELACIONADOS CON LA ENSEÑANZA Y EL APRENDIZAJE DE LAS GEOCIENCIAS.}

educativos de la Comunidad Autónoma de Extremadura (España). Esta autoevaluación ha requerido el diseño de un cuestionario acerca de la intervención educativa fuera del aula dirigido al alumnado y profesorado. Fruto de esta experiencia se realizan propuestas de mejora en las estrategias para las intervenciones educativas fuera de las aulas.

Palabras-clave: intervenciones educativas, estrategia de investigación, innovación, estudio de caso, COVID-19.

\section{ABSTRACT}

For just over a year, the COVID-19 pandemic has been producing a global impact that has had a special effect on health and educational matters. In countries like Spain, the teaching and learning processes have been affected all over educational levels.

In this extraordinary situation, the Spanish education system has tried to adapt itself to the new situation characterized by restrictions to attenuate the effects of the pandemic, sometimes promoting novel teaching strategies, such as the almost exclusive use of ICT in a situation of exceptional during the middle of the course. In those scientific disciplines where outdoor classrooms are common, however, there have also been constant changes in work procedures, which are also necessary to adapt teaching to the new status quo.

In some scientific disciplines where the use of laboratories and outdoor classrooms is usual, the pandemic was particularly affected during the 2019-20 academic year. However, during the last year, special teaching measures with adequate ventilation have led the outdoor classrooms in subjects related to Biology and Geology, based on educational interventions in the environment.

The result has been an image that allows us to present in this work the comparison of the data obtained after analyzing the impressions on the participation of 648 male and female students from eighteen educational centers in the Autonomous Community of Extremadura (Spain). This self-evaluation required the design of a questionnaire about educational intervention outdoor classroom aimed at students and teachers. As a result of this experience, proposals are made to improve strategies for educational interventions outdoor classroom.

Key-words: educational interventions, research strategy, novelty, case study, COVID-19.

\section{INTRODUCCIÓN}

Como señalan Moraza y Antón (2014), "Las decisiones que adopta el investigador cuando intenta entender y explicar un fenómeno tienen siempre consecuencias". Podemos añadir además que la recogida de los datos con los que abordar un análisis de una experiencia profesional suele tener a menudo carácter subjetivo.

Siguiendo a estos mismos autores, a la hora de proponer un objetivo educativo específico, un investigador puede fácilmente plantearse una serie de cuestiones, a modo de reflexiones sobre su papel en la propia investigación: lo que significa enseñar, aprender y evaluar; el valor de la evaluación en sí misma y aplicada a la materia en concreto (en este caso, las Geociencias), así como si la propia experiencia modifica la práctica docente. También puede plantearse la necesidad de establecer criterios objetivos de evaluación y si estos deben se abiertos y públicos, etc. Además, sus percepciones y emociones pueden alterar tanto su comportamiento como el resultado de la intervención que lleve a cabo con el alumnado (Mellado et al., 2012).

Siendo conscientes del sesgo que puede modelar la subjetividad del investigador, se hace necesario estandarizar el procedimiento de adquisición de datos, a modo de cuestionario o ficha, para que resulte útil y dar cierto grado de objetividad a las conclusiones. Es conocido que las decisiones adoptadas por el investigador en el diseño metodológico y en la puesta en marcha de su proyecto educativo afecta al resultado obtenido. Quizá por ello resulte procedente señalar que lo que pretende demostrar en este estudio es el camino seguido por si fuera de utilidad a otros investigadores, pudiendo ser especialmente aplicable en periodos de crisis, dadas las dificultades añadidas a un sistema educativo ya de por sí afectado por la difícil adaptabilidad a determinadas prácticas educativas y situaciones críticas, como la vivida debido a la pandemia COVID-19.

Woodbury y Gess-Newsome (2002), Jay y Johnson (2002), Lotter y Miller (2017) y más recientemente Vázquez-Bernal et al. (2019) plantean que el modo en que el profesorado piensa está determinado por la interde- 
pendencia de las facetas del contexto general en el que llevan a cabo su práctica docente, así como en la forma y la profundidad en que la reflexión se enfoca. Por otro lado, dado que la propia conducta y experiencia vital de un investigador influirá en sus concepciones (Schraw y Olafson, 2003) parece fundamental intentar cuantificar las valoraciones subjetivas, en un intento, sólo eso, de acercar la teoría a la investigación educativa (Antón y Moraza, 2014) con la idea de autoeficacia de fondo (Brígido et al, 2014). Aunque aplicable al caso del cambio de modelo educativo, parece oportuno citar a Reza (2019), autora que plantea el interés en analizar la variedad de sentimientos y acciones que dicho cambio produce en los docentes, en cuanto a curiosidad, incertidumbres, investigación y estudio para su adecuado manejo y dominio, y los discentes, en cuanto a las estrategias y actividades que realizan bajo la planificación, dirección y guía del docente, para que sean adecuadas y conduzcan a los propósitos que se pretenden, lo cual lleva a la práctica reflexiva permanente que tiene como objetivo la transformación del ejercicio docente para ofrecer al alumnado un servicio de mayor calidad.

El marco de nuestra investigación incluye un proceso teórico-práctico, que hemos denominado intervención educativa, consistente en una estrategia que contempla actividades dentro y fuera de las aulas, para la materia de Ciencias Naturales, habiéndose aplicado concretamente para la enseñanza y el aprendizaje de las Ciencias Geológicas o Geociencias. Este tipo de intervenciones educativas no son nuevas, sin embargo, lo que diferencia a este modelo de otros es que las salidas fuera del aula se realizan en entornos urbanos próximos, cuando lo habitual es que dichas salidas utilicen el medio natural como contexto para la enseñanza de conceptos geocientíficos (formación y reciclaje de rocas y minerales, fenómenos sedimentológicos y tectónicos, etc.). La investigación utiliza como propuesta geográfica un Bien de Interés Cultural denominado Vía de la Plata (figura 1) y reconocido como Sitio Histórico, que recorre Extremadura de sur a norte a lo largo de 300 kilómetros, y que atraviesa numerosas poblaciones donde es común encontrar elementos patrimoniales (Maldonado et al., 2018) que sirven de apoyo a la docencia de las Geociencias de un modo particular, teórico-práctico (Rebollada et al., 2018).

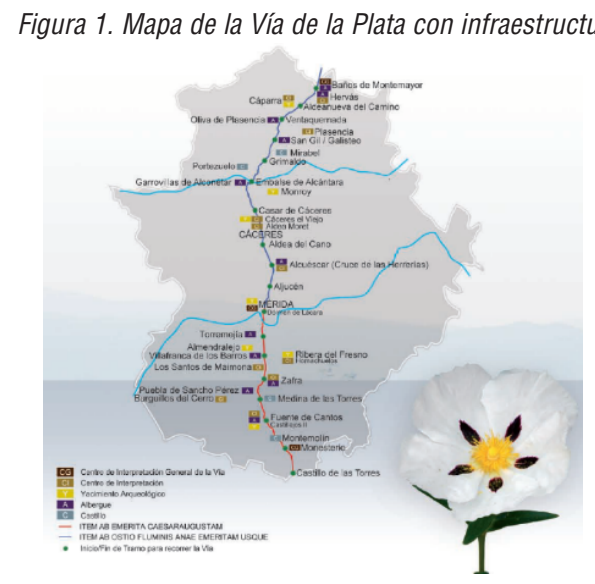

Elementos del patrimonio histórico, artístico, arqueológico, industrial y natural se conjugan en prácticamente todas las poblaciones que atraviesa dicho corredor cultural, permitiendo utilizar, desde un punto de vista didáctico, las rocas con las que están construidos diversos monumentos, los museos y centros de interpretación, los paisajes o el relieve, o el uso industrial de minerales y rocas, entre otros, para incorporar al proceso de enseñanza de las Geociencias prácticamente todo el elenco de conceptos básicos en el currículo de dicha materia en la Enseñanza Secundaria (tabla 1). 
Tabla 1.- Elementos del patrimonio utilizados para la enseñanza y el aprendizaje de las Geociencias.

\begin{tabular}{|c|c|c|c|c|c|c|}
\hline \multirow[b]{2}{*}{$\begin{array}{c}\text { RUTAS } \\
\text { REALIZADAS }\end{array}$} & \multicolumn{6}{|c|}{ RECURSOS DIDÁCTICOS UTILIZADOS } \\
\hline & 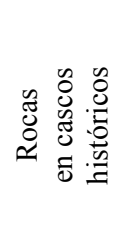 & 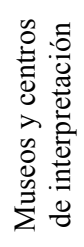 & 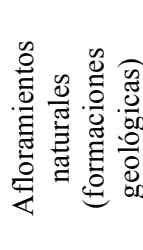 & 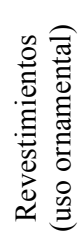 & 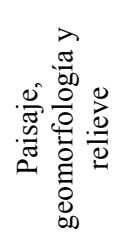 & 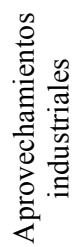 \\
\hline Hervás & $\mathrm{X}$ & $\mathrm{X}$ & & $\mathrm{X}$ & $\mathrm{X}$ & \\
\hline $\begin{array}{l}\text { Zarza de } \\
\text { Granadilla }\end{array}$ & $\mathrm{X}$ & & & $\mathrm{X}$ & $\mathrm{X}$ & \\
\hline Plasencia & $\mathrm{X}$ & $\mathrm{X}$ & $\mathrm{X}$ & $X$ & $\mathrm{X}$ & \\
\hline Galisteo & $\mathrm{X}$ & $\mathrm{X}$ & $\mathrm{X}$ & $\mathrm{X}$ & $\mathrm{X}$ & \\
\hline Garrovillas & $\mathrm{X}$ & $\mathrm{X}$ & & $\mathrm{X}$ & $\mathrm{X}$ & \\
\hline Casar de Cáceres & $\mathrm{X}$ & $\mathrm{X}$ & & & $\mathrm{X}$ & \\
\hline Cáceres & $\mathrm{X}$ & $\mathrm{X}$ & $\mathrm{X}$ & $\mathrm{X}$ & $\mathrm{X}$ & $\mathrm{X}$ \\
\hline Montánchez & $\mathrm{X}$ & & & $\mathrm{X}$ & $\mathrm{X}$ & \\
\hline Alcuéscar & $\mathrm{X}$ & & & $\mathrm{X}$ & $\mathrm{X}$ & \\
\hline Mérida & $\mathrm{X}$ & $\mathrm{X}$ & & & $\mathrm{X}$ & \\
\hline Almendralejo & $\mathrm{X}$ & $\mathrm{X}$ & & $\mathrm{X}$ & $\mathrm{X}$ & \\
\hline $\begin{array}{l}\text { Villafranca de los } \\
\text { Barros }\end{array}$ & $\mathrm{X}$ & & & $\mathrm{X}$ & $\mathrm{X}$ & \\
\hline $\begin{array}{l}\text { Los Santos de } \\
\text { Maimona }\end{array}$ & $\mathrm{X}$ & $\mathrm{X}$ & $\mathrm{X}$ & & $\mathrm{X}$ & $\mathrm{X}$ \\
\hline Zafra & $\mathrm{X}$ & $\mathrm{X}$ & & $\mathrm{X}$ & & \\
\hline Fuente de Cantos & $\mathrm{X}$ & & & $\mathrm{X}$ & $\mathrm{X}$ & \\
\hline Monesterio & $\mathrm{X}$ & & & $\mathrm{X}$ & $\mathrm{X}$ & \\
\hline
\end{tabular}

Entre los numerosos autores que han estudiado la epistemología de las Geociencias, destacan David Brusi, Emilio Pedrinaci y Amelia Calonge, además de su habitual equipo de investigadores colaboradores. El primero ha analizado en numerosas ocasiones las salidas de campo, en su sentido habitual, concluyendo la necesidad de una planificación si cabe mayor que la de una clase ordinaria (Brusi, 1992), haciendo especial hincapié en las competencias (Brusi et al., 2011), puesto que no hacerlo supone no aprovechar al máximo un tipo de actividad en la que se invierten numerosos recursos personales y del propio sistema educativo. Pedrinaci aborda más transversalmente la enseñanza de las Geociencias, destacando en temas curriculares relacionados con el conocimiento específico del contenido de la materia, como las ideas-clave sobre las que pivota su enseñanza y aprendizaje, en lo que se ha denominado Alfabetización en Ciencias de la Tierra (Pedrinaci, 2013). Amelia Calonge y colaboradores, por otro lado, han profundizado en las rutas geológicas como medio para la enseñanza de las Geociencias, culminando en el proyecto GEOschools para Enseñanza Secundaria Obligatoria (Calonge et al., 2014).

\section{OBJETIVOS}

Este trabajo podría enmarcarse dentro de la psicología positiva que afirma aquello de: "Donde hay un problema debe haber una solución". La investigación educativa se realizó modo de estudio de caso, y durante la misma se incluyó un análisis subjetivo interno de la propia intervención educativa. Se enmarcaba todo ello en 
una investigación de mayor alcance, consistente en el estudio de las modificaciones del proceso de enseñanzaaprendizaje de las Geociencias en alumnos de $3^{0}$ y $4^{0}$ de ESO en la Comunidad Autónoma de Extremadura (España).

El objetivo principal de este trabajo es presentar el procedimiento específico de autoevaluación (y/o de reflexión), de las capacidades cognitivas y de actitudes y habilidades del alumnado y profesorado de apoyo en el marco de diferentes intervenciones educativas realizadas fuera de las aulas, con la intención de utilizar dicho procedimiento en determinadas circunstancias desfavorables a la práctica docente (falta de medios y dificultades propias del sistema educativo en sí y de los centros educativos en particular).

El procedimiento llevado a cabo se encuadra en lo que podríamos denominar un estudio de caso, puesto que el investigador decide realizar una autoevaluación de su desempeño, no mediante una evaluación de su propio trabajo, sino de las experiencias, sensaciones y percepciones que recibe durante las intervenciones educativas en las que participa como director.

Esta pretendida retroalimentación es planteada mediante el análisis de varios aspectos que giran en torno a las intervenciones educativas fuera de las aulas y sobre la posible mejora de los conocimientos que los alumnos y alumnas obtendrían hipotéticamente durante las salidas fuera de las aulas y que estarían focalizadas en distintas cuestiones geocientíficas.

A los conocimientos adquiridos se unen otros aspectos no menos importantes, según la práctica educativa, como son las actitudes y las habilidades que demuestra el alumnado, muy relacionados con la eficacia del aprendizaje.

Finalmente, la asistencia y apoyo del profesorado son evaluados por el investigador de una manera algo más objetiva, debido especialmente a que se trata de un número de participantes sensiblemente inferior, lo que facilita la toma de datos y posterior evaluación.

A fin de conocer el alcance y grado de validez intrínseca del procedimiento de autoevaluación seguido, toda la información recabada, en especial el aprendizaje cognitivo producido en alumnos y alumnas tras la realización de salidas fuera del aula, se ha contrastado con las conclusiones preliminares de la investigación en la que se encuadra la intervención educativa, que se resumen en una adquisición de conceptos geocientíficos genérica, así como una mejora de habilidades y actitudes.

\section{MÉTODO}

El diseño del procedimiento de investigación desarrollado con los centros educativos involucrados incluía una intervención fuera del aula con los alumnos. La intervención contempla, a su vez, una fase previa y otra posterior de evaluación de conocimientos, a fin de conocer la variabilidad producida por la propia salida fuera del aula. Esta se realizaba en el entorno del centro educativo, buscando facilitar la labor del profesorado a la hora de su planificación, así como evitar los problemas inherentes que las salidas generan en la organización de los centros educativos, además de los riesgos que conllevan.

Además de los cuestionarios cognitivos (figura 2) aplicados para evaluar la evolución de los conocimientos en Geociencias entre la fase previa y la posterior a la salida, se diseñaron materiales educativos específicos, como una guía gráfica para la docencia y diversos mapas conceptuales. 


\section{EL MEDIO COMO ESPACIO EDUCATIVO EN TIEMPOS DE CRISIS: EVALUACIÓN DE UNA EXPERIENCIA FUERA DEL AULA Y SU APLICACION, LA TRANSMISION DE ACTITUDES, VALORES Y CONOCIMIENTOS RELACIONADOS CON LA ENSEÑANZA Y EL APRENDIZAJE DE LAS GEOCIENCIAS.}

Figura 2. Cuestionario utilizado para evaluar la adquisición y/o mejora de conocimientos geocientíficos.
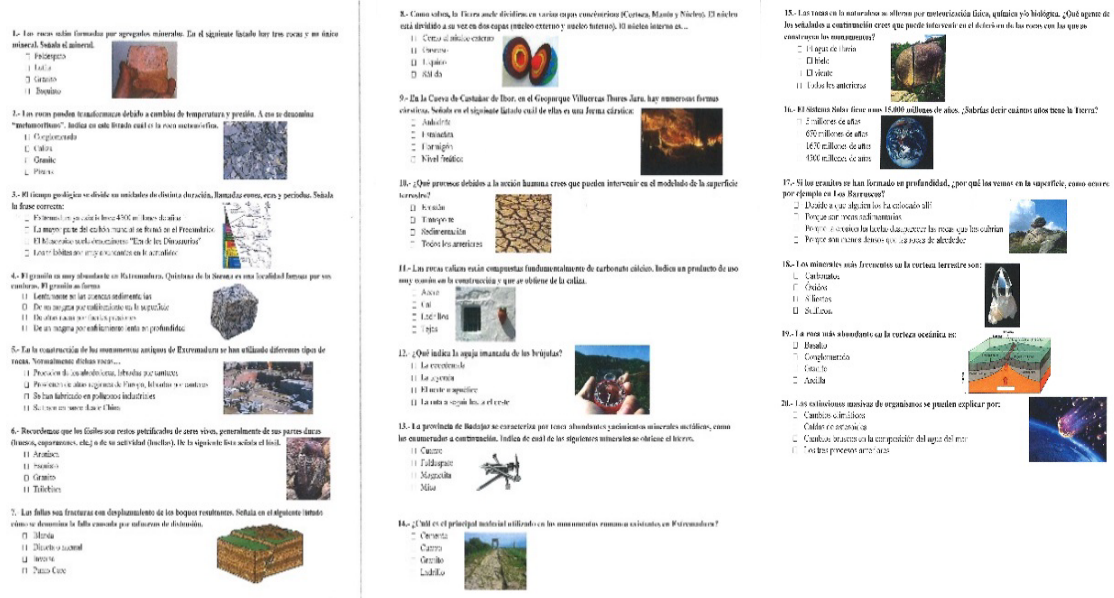

Los mapas conceptuales (figura 3) en concreto constituyen una herramienta didáctica de enorme valor. Ideados originalmente por Joseph Novak en 1977 en la Universidad de Cornell, son hoy en día un instrumento muy utilizado, gracias a la plataforma en abierto del Institute for Human and Machine Cognition, de Pensacola, Florida (EE.UU.), https://cmap.ihmc.us/, desde donde se puede descargar la aplicación con la que crear mapas conceptuales personalizados a la materia y al nivel educativo deseados.

Figura 3. Ejemplo de mapa conceptual diseñado para la intervención educativa fuera del aula.

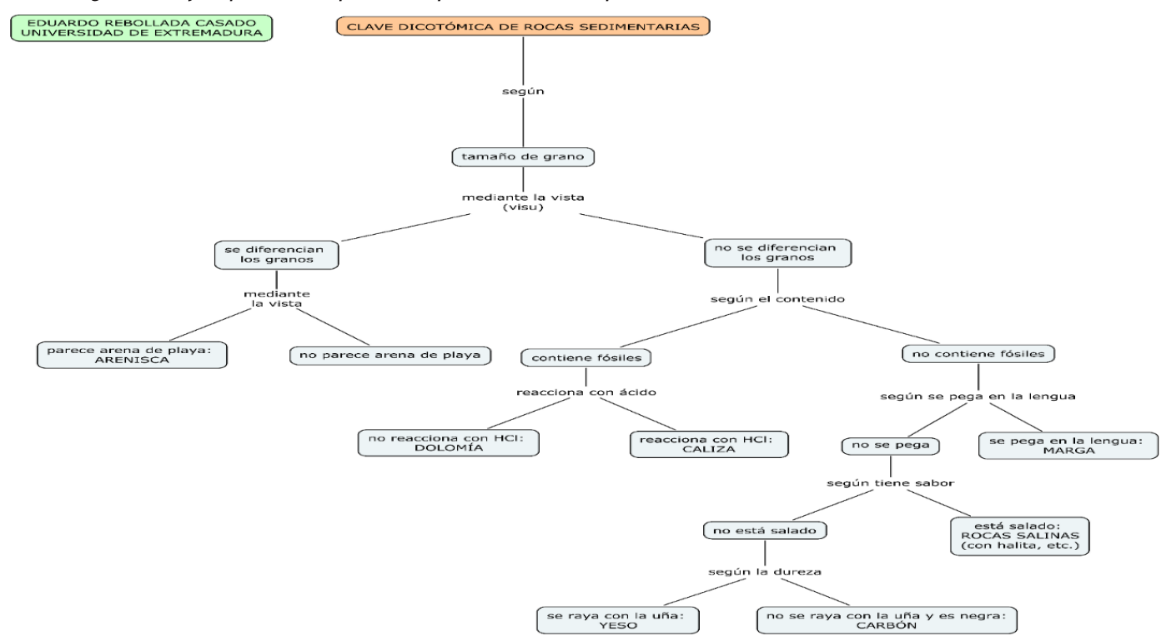

El investigador principal, por su experiencia docente, se marcó un guion de trabajo, que no obstante era flexible para adaptarlo a las circunstancias que rodeaban a cada salida fuera del aula (tiempo, recorrido, meteorología, número de alumnos, número de profesores colaboradores, etc.). 
El proceso de enseñanza giraba en torno a ocho ideas que sintetizan los conceptos fundamentales del currículo geocientífico en niveles de $3^{\circ}$ y $4^{0}$ de ESO, de acuerdo a la normativa vigente: tiempo geológico, estructura de la Tierra, evolución, ciclo de las rocas, tectónica de placas, modelado, materiales y riesgos. Dichas ideas fundamentales fueron la base de las explicaciones, enlazándolas con los elementos del patrimonio histórico-artístico, industrial o natural visitado.

En el marco de intervención, claramente intencional, no sólo por los niveles educativos formales elegidos ( $3^{\circ}$ y $4^{0}$ de ESO), sino también por los centros educativos seleccionados, localizados cerca de elementos del patrimonio, la salida fuera del aula, a modo de salida de campo, era la actividad principal, sobre la que giraban el resto de las acciones de enseñanza y aprendizaje.

Las principales conclusiones a las que hemos llegado con esta investigación son:

1.- El valor determinante de la planificación de las intervenciones educativas para el éxito de las salidas fuera del aula.

2.- El enorme valor didáctico que pueden llegar a tener los elementos patrimoniales existentes en las áreas urbanas.

3.- La posibilidad de una mejora en los conocimientos geocientíficos adquiridos por el alumnado tras las salidas fuera del aula por los entornos urbanos. Esta mejora es mayor en el alumnado de $3^{\circ}$ de ESO en comparación con el de 4 , debido a que estos últimos parten con la ventaja de partida de disponer de una mayor base curricular geocientífica (han superado el curso $3^{0}$ de ESO).

4.- La mejora aún mayor apreciada en aquellos alumnos y alumnas que partían de un menor nivel de conocimientos, denotando la importancia de la salida de campo para la enseñanza y el aprendizaje eficaces de la materia de Geociencias.

\section{PARTICIPANTES}

La muestra, cuya composición de detalla en la tabla 2, sobre la que se llevó a cabo la investigación fue de 648 alumnos, repartidos en 18 centros educativos públicos de Enseñanza Secundaria de 14 localidades de Extremadura (España).

Además, participaron 29 docentes de apoyo, variando entre 1 y 3 el número de docentes por cada centro educativo participante. 
EL MEDIO COMO ESPACIO EDUCATIVO EN TIEMPOS DE CRISIS: EVALUACIÓN DE UNA EXPERIENCIA FUERA DEL AULA Y SU APLICACION, LA TRANSMISION DE ACTITUDES, VALORES Y CONOCIMIENTOS RELACIONADOS CON LA ENSEÑANZA Y EL APRENDIZAJE DE LAS GEOCIENCIAS.

Tabla 2. Alumnado participante en la investigación, por género, curso y localidad de sus respectivos centros educativos.

\begin{tabular}{|l|c|c|c|c|c|c|}
\hline $\begin{array}{l}\text { Localidad } \\
\text { del centro } \\
\text { educativo }\end{array}$ & Centros educativos & Alumnos & Alumnas & $\begin{array}{c}\mathbf{3}^{\mathbf{0}} \\
\text { ESO }\end{array}$ & $\begin{array}{c}\mathbf{4}^{\mathbf{o}} \\
\text { ESO }\end{array}$ & $\begin{array}{c}\text { Docentes } \\
\text { de apoyo }\end{array}$ \\
\hline Hervás & Valle del Ambroz & 9 & 9 & 0 & 18 & 1 \\
\hline $\begin{array}{l}\text { Zarza de } \\
\text { Granadilla }\end{array}$ & Cáparra & 5 & 4 & 0 & 9 & 1 \\
\hline Plasencia & Gabriel y Galán & 18 & 25 & 18 & 25 & 2 \\
\hline Galisteo & Galisteo & 21 & 26 & 36 & 11 & 2 \\
\hline $\begin{array}{l}\text { Garrovillas } \\
\text { de Alconétar }\end{array}$ & Alconétar & 14 & 20 & 25 & 9 & 1 \\
\hline $\begin{array}{l}\text { Casar de } \\
\text { Cáceres }\end{array}$ & Vía de la Plata & 4 & 6 & 0 & 10 & 1 \\
\hline \multirow{2}{*}{ Cáceres } & Eniversidad Laboral & 7 & 12 & 19 & 0 & 2 \\
\cline { 2 - 7 } & El Brocense & 25 & 25 & 30 & 20 & 1 \\
\cline { 2 - 7 } & Norba Caesarina & 31 & 25 & 17 & 39 & 2 \\
\cline { 2 - 7 } & Al-Qázeres & 6 & 11 & 0 & 17 & 1 \\
\hline Morcía Téllez & 27 & 34 & 33 & 28 & 2 \\
\hline Alcuéscar & Santa Lucia del Trampal & 31 & 35 & 42 & 24 & 2 \\
\hline Mérida & Albarregas & 8 & 17 & 0 & 25 & 2 \\
\hline Almendralejo & Santiago Apóstol & 19 & 8 & 0 & 27 & 1 \\
\hline Zafra & Suárez de Figueroa & 20 & 15 & 0 & 35 & 3 \\
\hline $\begin{array}{l}\text { Fuente de } \\
\text { Cantos }\end{array}$ & Vía de la Plata & 30 & 10 & 0 & 40 & 1 \\
\hline Monesterio & Maestro Juan Calero & 32 & 33 & 36 & 29 & 2 \\
\hline \multirow{2}{*yyyyyn}{} & 321 & 327 & 266 & 382 & 29 \\
\cline { 2 - 6 } & & 648 & 648 & 16 & 2 \\
\hline
\end{tabular}

Los dieciocho centros educativos de secundaria tienen en común ubicarse en poblaciones con elementos patrimoniales útiles para el desarrollo de actividades fuera de las aulas. Estos elementos pueden ser desde construcciones con valor histórico-artístico y arquitectónico (palacios, iglesias, fuentes, puentes, acueductos...) hasta elementos de arquitectura industrial o restos de actividades industriales relacionadas con la minería, pasando por museos y parques, lugares donde es posible encontrar recursos educativos de origen natural. Los elementos utilizados por el investigador para focalizar la salida, con itinerarios diseñados a tal fin, se han enumerado en la tabla 1.

\section{INSTRUMENTO}

Se ha diseñado una ficha de recogida de datos post-intervención fuera del aula, que era cumplimentada una vez finalizada la visita (figura 4), y en la que se incluyen aspectos referidos tanto al alumnado como al profesorado.

Con la ficha y el análisis posterior, se obtuvo una serie de datos de interés para contrastar con el objetivo fundamental de la investigación, saber si mejoraría el grado de adquisición de conocimientos gracias a realizar una salida fuera del aula para estudiar directa o indirectamente elementos geocientíficos. 
El evaluador que participa en la intervención analiza las circunstancias que rodean a cada grupo de alumnos y profesores durante las salidas.

Figura 4. Ficha diseñada y utilizada por el investigador, para documentar sus experiencias y percepciones tras la realización de las salidas fuera del aula.

Encuesta del investigador al grupo de alumnos y profesores, sobre diferentes aspectos trabajados IES Fecha

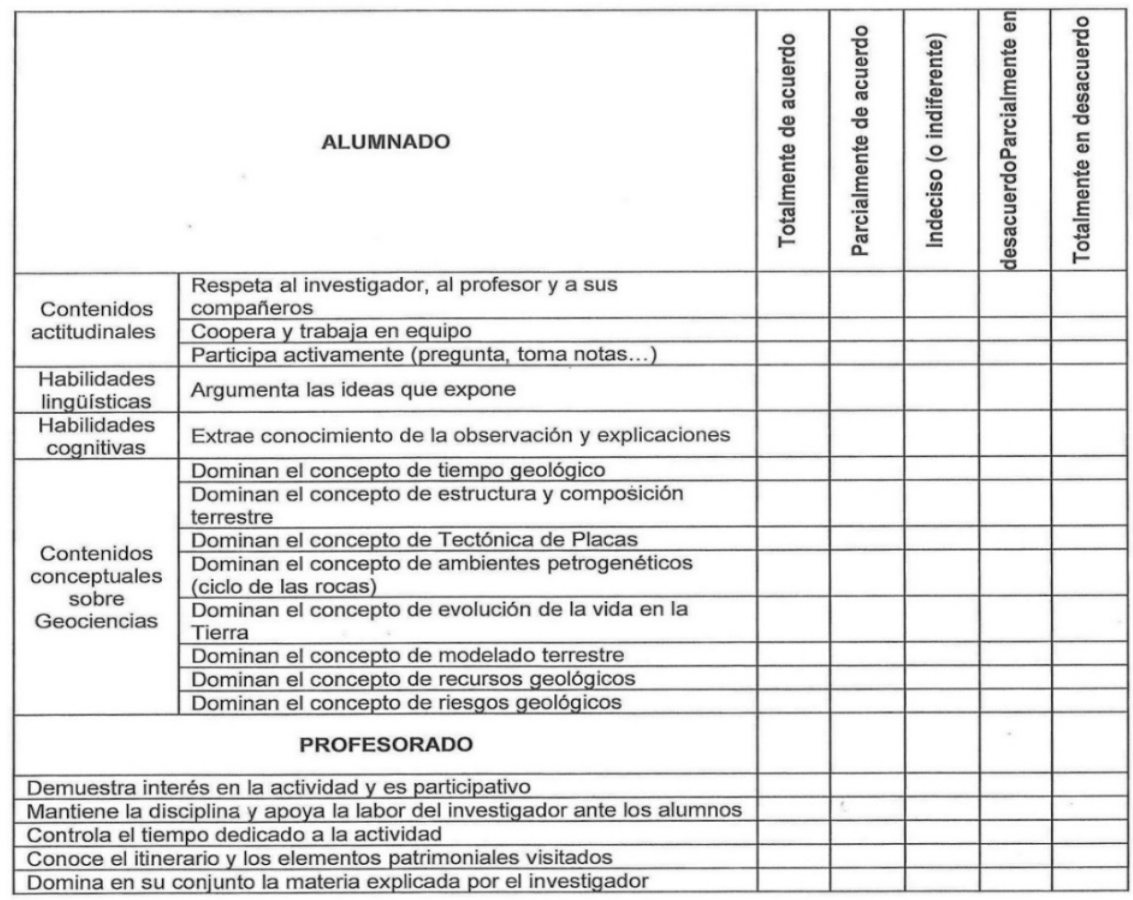

La ficha se compone de dos partes: una, correspondiente a los alumnos y otra para el profesorado.

La primera parte tiene en cuenta tres apartados fundamentales: actitudes, habilidades y conocimientos. Las habilidades, a su vez, se subdiven en habilidades lingüísticas y cognitivas.

Los contenidos actitudinales incluyen tres cuestiones consideradas fundamentales en la realización de una salida fuera del aula: el respeto hacia las demás personas, tanto las que componen el grupo como terceros; el trabajo en equipo, muy importante al realizarse tareas en grupo; la participación activa, una muestra de su interés y atención hacia el guía/investigador.

Las habilidades lingüísticas son una forma de percibir la capacidad y competencia argumental y de expresión general, valorándose el manejo de la terminología específica propia de la materia (teorías, términos geológicos y sus definiciones, etc.).

Las habilidades cognitivas evalúan la percepción sobre la adquisición y manejo de conceptos, quizá la parte más subjetiva de la ficha, por la dificultad de aproximación a dicha realidad. Para ello se utilizó como referencia la simbología de la propia obtención de conocimiento por parte del alumnado. Esta adquisición y/o mejora de conocimientos geocientíficos está claramente relacionada con las otras habilidades y, de fondo, con la actitud general durante la salida. 


\section{EL MEDIO COMO ESPACIO EDUCATIVO EN TIEMPOS DE CRISIS: EVALUACIÓN DE UNA EXPERIENCIA FUERA DEL AULA Y SU APLICACION, LA TRANSMISION DE ACTITUDES, VALORES Y CONOCIMIENTOS RELACIONADOS CON LA ENSEÑANZA Y EL APRENDIZAJE DE LAS GEOCIENCIAS.}

Por último, los contenidos conceptuales están muy relacionados con la adquisición y/o mejora de conocimientos, interesa por representar para el observador la percepción que tiene de su labor docente, como director de la actividad fuera del aula.

La parte de la ficha del profesorado, por otro lado, incluye cuestiones que indagan sobre su interés en la actividad y su participación y/o implicación en la misma.

\section{RESULTADOS}

El análisis de los datos se presenta en relación con las actitudes, las habilidades y los contenidos conceptuales del alumnado, así como de las actitudes del profesorado.

1.- Actitudes del alumnado (tabla 3):

Las actitudes de los diferentes grupos de alumnos y alumnas durante las salidas fuera de las aulas son variables, a los ojos del observador.

Se observa que el 50\% (9 salidas fuera del aula) de la muestra adopta una postura de respeto hacia sus compañeros, sus profesores y el propio investigador. El resto de la muestra analizada presenta variabilidad, aunque en general se acercan más hacia el respeto que hacia todo lo contrario.

No ocurre lo mismo para la cooperación y el trabajo en equipo, que no parece ser una actitud habitual entre el alumnado, a la luz de los datos que desprende la ficha: menos de la mitad parecen trabajar en equipo, aunque no haya una tendencia clara a no realizarlo si fuera necesario.

Más dispersión de datos, si cabe, se produce para el siguiente ítem, sobre la participación activa durante la actividad. A tendencia es hacia la poca participación en la actividad, lo cual puede deberse a que no existe suficiente confianza entre el alumnado y el investigador, fundamental para que exista fluidez en el diálogo y la relación docente no sea solamente unidireccional (del investigador hacia los alumnos).

Tabla 3. Datos sobre las actitudes generales del alumnado.

\begin{tabular}{|c|c|c|c|c|c|}
\hline ACTITUDES & 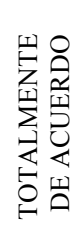 & 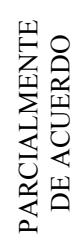 & 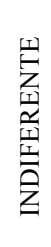 & 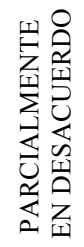 & 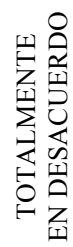 \\
\hline $\begin{array}{l}\text { Respeta al investigador, al } \\
\text { profesor y a sus compañeros }\end{array}$ & 4 & 9 & 2 & 3 & 0 \\
\hline Coopera y trabaja en equipo & 0 & 7 & 8 & 3 & 0 \\
\hline $\begin{array}{l}\text { Participa activamente (pregunta, } \\
\text { toma notas...) }\end{array}$ & 2 & 4 & 7 & 5 & 0 \\
\hline
\end{tabular}

2.- Habilidades lingüísticas (tabla 4):

Estas se relacionan con la competencia lingüística y con el manejo adecuado de cierta terminología científica. Según los datos obtenidos, no existe una clara tendencia ni positiva ni negativa en este asunto, aunque en términos generales el sesgo estaría quizá un poco desplazado hacia un correcto uso de la argumentación geológica. 
Tabla 4. Datos sobre las habilidades lingüísticas del alumnado

\begin{tabular}{|c|c|c|c|c|c|}
\hline $\begin{array}{l}\text { HABILIDADES } \\
\text { LINGÜÍSTICAS }\end{array}$ & 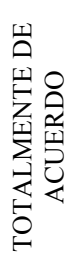 & 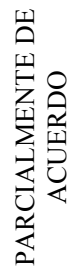 & 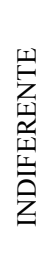 & 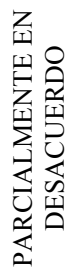 & 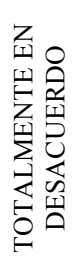 \\
\hline $\begin{array}{l}\text { Argumenta las ideas } \\
\text { que expone }\end{array}$ & 2 & 7 & 7 & 2 & 0 \\
\hline
\end{tabular}

3.- Habilidades cognitivas (tabla 5):

Como se ha indicado, las habilidades cognitivas son fundamentales en la investigación-marco, al ser el aprendizaje de conceptos el parámetro evaluado mediante la diferencia entre el antes y el después a la salida fuera del aula.

Tabla 5. Datos sobre las habilidades cognitivas del alumnado

\begin{tabular}{|c|c|c|c|c|c|}
\hline $\begin{array}{l}\text { HABILIDADES } \\
\text { COGNITIVAS }\end{array}$ & 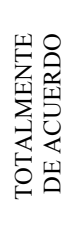 & 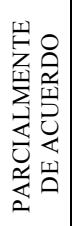 & 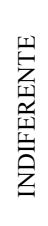 & 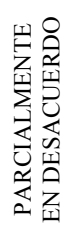 & 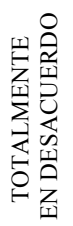 \\
\hline $\begin{array}{l}\text { Extrae conocimiento } \\
\text { de la observación y } \\
\text { explicaciones }\end{array}$ & 1 & 10 & 6 & 1 & 0 \\
\hline
\end{tabular}

4.- Contenidos conceptuales (tabla 6):

Respecto a los contenidos sobre Geociencias, las ocho ideas clave sobre las que se ha indagado apuntan a que el alumnado presenta de manera general un dominio de algunas de ellas, aunque este no sea pleno. Es el caso de los conceptos sobre tiempo geológico, estructura y composición terrestre y recursos geológicos.

En algunas de las ideas, como la Tectónica de Placas, sí parece que hay una idea más clara sobre su significado.

Sin embargo, en prácticamente la mitad de las ideas (ciclo de las rocas, evolución de la vida en la Tierra, modelado terrestre y riesgos geológicos) el alumnado no demuestra un dominio evidenciado de los conceptos con aquellas relacionados. 


\section{EL MEDIO COMO ESPACIO EDUCATIVO EN TIEMPOS DE CRISIS: EVALUACIÓN DE UNA EXPERIENCIA FUERA DEL AULA Y SU APLICACION, LA TRANSMISION DE ACTITUDES, VALORES Y CONOCIMIENTOS RELACIONADOS CON LA ENSEÑANZA Y EL APRENDIZAJE DE LAS GEOCIENCIAS.}

Tabla 6. Datos sobre la capacidad del alumnado respecto a determinados contenidos.

\begin{tabular}{|c|c|c|c|c|c|}
\hline $\begin{array}{c}\text { CONTENIDOS CONCEPTUALES SOBRE } \\
\text { GEOCIENCIAS }\end{array}$ & 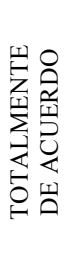 & 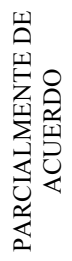 & 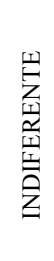 & 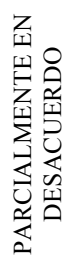 & 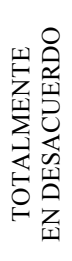 \\
\hline Dominan el concepto de tiempo geológico & 1 & 8 & 8 & 1 & 0 \\
\hline $\begin{array}{l}\text { Dominan el concepto de estructura y } \\
\text { composición terrestre }\end{array}$ & 1 & 8 & 8 & 1 & 0 \\
\hline Dominan el concepto de Tectónica de Placas & 3 & 6 & 8 & 1 & 0 \\
\hline Dominan el concepto del ciclo de las rocas & 0 & 5 & 10 & 2 & 1 \\
\hline $\begin{array}{l}\text { Dominan el concepto de evolución de la vida } \\
\text { en la Tierra }\end{array}$ & 0 & 7 & 10 & 1 & 0 \\
\hline Dominan el concepto del modelado terrestre & 0 & 6 & 10 & 2 & 0 \\
\hline Dominan el concepto de recursos geológicos & 0 & 9 & 7 & 2 & 0 \\
\hline Dominan el concepto de riesgos geológicos & 0 & 7 & 10 & 1 & 0 \\
\hline
\end{tabular}

5.- En cuanto a los resultados del cuestionario referidos al profesorado, se observa lo siguiente (tabla 7):

Una neta participación e interés, demostrable por la planificación y organización, la anotación en la agenda docente de la actividad, así como la definición de los roles tanto del investigador como del alumnado y el suyo propio como parte del equipo docente del centro educativo.

En concreto, se observa una clara implicación en la misma, demostrable por el papel jugado en el mantenimiento de la disciplina durante las salidas, el control de tiempos y apoyo a la labor investigadora.

Finalmente, demuestra un control claro y explícito de la materia, aspecto muy útil para abordar este tipo de experiencias.

Tabla 7. Datos sobre los equipos docentes (habilidades y actitudes).

\begin{tabular}{|c|c|c|c|c|c|}
\hline PROFESORADO & 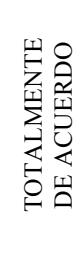 & 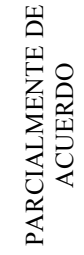 & 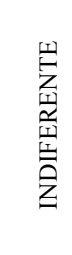 & 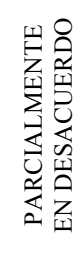 & 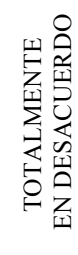 \\
\hline $\begin{array}{l}\text { Demuestra interés en la actividad y es } \\
\text { participativo }\end{array}$ & 13 & 5 & 0 & 0 & 0 \\
\hline $\begin{array}{l}\text { Mantiene la disciplina y apoya la labor del } \\
\text { investigador ante los alumnos }\end{array}$ & 16 & 1 & 1 & 0 & 0 \\
\hline Controla el tiempo dedicado a la actividad & 16 & 1 & 1 & 0 & 0 \\
\hline $\begin{array}{l}\text { Conoce el itinerario y los elementos } \\
\text { patrimoniales visitados }\end{array}$ & 11 & 4 & 2 & 0 & 1 \\
\hline $\begin{array}{l}\text { Domina en su conjunto a materia explicada } \\
\text { por el investigador }\end{array}$ & 16 & 0 & 2 & 0 & 0 \\
\hline
\end{tabular}




\section{CONCLUSIONES}

Siguiendo el diseño propuesto creemos que esta experiencia puede resultar muy motivadora al profesorado que decida Ilevar a acabo salidas al medio, tanto al entorno urbano cercano como a espacios próximos a los centros educativos.

El modelo de intervención didáctica para la enseñanza de las Geociencias a través de salidas al entorno es extrapolable para la enseñanza de otras materias, como la Geografía, la Historia o cualquier otra realizando las consiguientes adaptaciones.

En tiempos de pandemia, como los que acabamos de vivir, donde los grupos han sufrido una limitación de movilidad y un confinamiento, hemos podido aprender en salidas al medio que, a pesar de las mascarillas, hemos disfrutado del entorno y de la libertad de sentirnos seguros en espacios abiertos.

La evaluación de la experiencia ha sido altamente positiva. Nos ha hecho descubrir algunos aspectos que requieren ser ajustados y fortalezas que debemos potenciar en próximas intervenciones.

Afortunadamente, vivimos en espacios que poseen un patrimonio natural y/o cultural que facilitan la realización de salidas fuera del aula al medio cercano a nuestros centros educativos, que resultan motivadoras para los alumnos y atractivas para el profesorado, que ve en estas experiencias una forma de enseñanza distinta basada en el descubrimiento, la alfabetización en ciencias y capaz de fomentar el respeto, la cooperación y la participación activa.

\section{REFERENCIAS BIBLIOGRÁFICAS}

Antón, A., y Moraza, J.I. (2014). Concepciones de los docentes universitarios y evaluación de los aprendizajes. International Journal of Developmental and Educational Psychology, 1(5), 273-282.

Brígido, Mํㅗ., Borrachero, A.B., Bermejo, M. ․․, y Dávila, Mª A. (2014). Programa de intervención para la mejora de las creencias de autoeficacia en las clases de ciencias. International Journal of Developmental and Educational Psychology, 1(5), 73-80.

Brusi, D. (1992). Reflexiones en torno a la Didáctica de las salidas de campo en Geología l: Aspectos funcionales. II: Aspectos metodológicos. Actas VII Simposio sobre Enseñanza de la Geología. Santiago de Compostela, 363-407.

Brusi, D., Zamorano M., Casellas R., y Bach, J. (2011). Reflexiones sobre el diseño por competencias en el trabajo de campo en Geología. Enseñanza de las Ciencias de la Tierra, 19 (1), 4-14.

Calonge, M… A., Fermeli, G., Meléndez, G., y Martínez, J.A. (2014). Proyecto GeoSchools: reflexiones sobre la Geología en la Enseñanza Secundaria Obligatoria. Geogaceta, 55, 99-102.

Maldonado, J.J., Corrales, J.Mํ.., y Cardenal, Mํ. M. (2018). Patrimonio natural, educativo, cultural y socioeconómico de la Vía de la Plata en Extremadura. En: Gómez-Ullate, M., Ochoa Siguencia, L., Álvarez-García, J. Del Río Rama, M. de la C. (ed.). Cultural Routes and Heritage. Tourism and Rural Development. Cultour +. Book of Proceedings: 53-74.

Mellado, V., Blanco, L.J., Borrachero, A.B., y Cárdenas, J.A. (2012). Las emociones en la enseñanza y el aprendizaje de las Ciencias y las Matemáticas (II). Grupo de Investigación DEPROFE. 328 p.

Moraza, J.I., y Antón, A. (2014). Las concepciones del docente universitario y la evaluación: génesis y desarrollo de la aplicación de un enfoque metodológico. International Journal of Developmental and Educational Psychology, 5(1), 261-272.

Jay, J., \& Johnson, K. (2002). Capturing complexity: a typology of reflective practice for teacher education. Teaching and Teacher Education, 18(1), 73-85.

Lotter, C.R., \& Miller, C. (2017). Improving inquiry teaching through reflection on practice. Research in Science education, 47(4), 913-942.

Pedrinaci, E. (2013). Alfabetización en Ciencias de la Tierra y competencia científica. Enseñanza de las Ciencias de la Tierra, 21(2), 208-214. 


\section{EL MEDIO COMO ESPACIO EDUCATIVO EN TIEMPOS DE CRISIS: EVALUACIÓN DE UNA EXPERIENCIA \\ FUERA DEL AULA Y SU APLICACION, LA TRANSMISION DE ACTITUDES, VALORES Y CONOCIMIENTOS \\ RELACIONADOS CON LA ENSEÑANZA Y EL APRENDIZAJE DE LAS GEOCIENCIAS.}

Rebollada, E., Corrales, J. Mª., y Rus, E. (2018). Patrimonio cultural de las ciudades vinculadas con la Vía de la Plata en Extremadura: un recurso para la enseñanza de las ciencias llevado a cabo en la ciudad de Cáceres. En: Gómez-Ullate, M., Ochoa Siguencia, L., Álvarez-García, J. Del Río Rama, M. de la C. (ed.). Cultural Routes and Heritage. Tourism and Rural Development. Cultour +. Book of Proceedings: 75-84.

Reza, J.K. (2019). La práctica reflexiva: requisito indispensable para poner en marcha el modelo educativo vigente. En: J.A. Trujillo Holguín, A.C. Ríos Castillo y J.L. García Leos (Coords.), Desarrollo profesional docente: reflexiones de maestros en servicio en el escenario de la Nueva Escuela Mexicana (pp. 103-114), Chihuahua, México: Escuela Normal Superior Prof. José E. Medrano R.

Schraw, G., y Olafson, L. (2003). Teacher's Epistemological World Views and Educational Practices. Journal of Cognitive Education and Psychology, 3(2), 178-235.

Vázquez-Bernal, B., Jiménez-Pérez, R., y Mellado, V. (2019). El conocimiento didáctico el contenido (CDC) de una profesora de ciencias: reflexión y acción como facilitadores del aprendizaje. Enseñanza de las Ciencias, 37(1), 25-53.

Woodbury, S., \& Gess-Newsome, J. (2002). Overcoming the paradox of change without difference: a model of change in the arena of fundamental school reform. Educational Policy, 16(5), 764-783. 